\title{
ISING MODEL ON TANGLED CHAIN. I. FREE ENERGY AND ENTROPY
}

\author{
R. MEJdani \\ International Centre for Theoretical Physics, Trieste 34100, Italy \\ and \\ Department of Pliysics, University of Tirana, Tirana, Albania*
}

(Reccived February 11, 1994)

\begin{abstract}
In this paper we have considered an Ising model defined on tangled chain, in which more bonds have been added to those of pure Ising chain. To understand their competition, particularly betwcen ferromagnetic and antiferromagnetic bonds, we have studied, using the transfer matrix method, some simple analytical calculations and an iterative algorithm, the behaviour of the free energy and entropy, particularly in the zero-field and zero-temperature limit, for different configurations of the ferromagnetic tangled chain and different types of additional intcractions (ferromagnetic or antiferromagnetic). We found that the condition $J=-J^{\prime}$ between the ferromagnetic interaction $J$ along the chain and the antiferromagnetic interaction $J^{\prime}$ across the chain is somewhat as a "transition region" condition for this behaviour. Our results indicate also the existence of non-zcro entropy at zero temperature.
\end{abstract}

PACS numbers: $65.50 .+\mathrm{m}, 75.10 .1 \mathrm{k}, 75.10 . \mathrm{Nr}, 05.50 .+\mathrm{q}$

\section{Introduction}

In [1] we have used a model of a lattice gas defined on a tangled chain to study the initial reaction-rate for the enzyme kinetics by a modified transfer matrix method, while in $[2,3]$, for Ising models on tangled chains or crumpled surfaces, the problem of metastable/unstable states, reflected in the equilibrium Gibbs average in terms of uneven effective local temperature is studied. In general, the existence of metastable statcs is responsible for some properties of spin glasses (see [4-6] and references cited therein). or random field magnets (see [7] and references cited therein). This is studied also for very simple models, namely

\footnotetext{
*Permanent address.
} 
for the random-nearest neighbour interaction Ising cliain [8] or the random-ficld Ising chain [9], where, always, an interplay between thermal and frustration effects is present. The frustration, as an important determining factor in the behaviour of interacting systems, since Toulouse first introduced the concept [10], refers to competition between different tcrms in IIamiltonian so that no spin configuration simultaneously minimizes each term. Some of the interactions of a considered spin with other ones could be positive, favoring parallel alignment, some negative, favoring antiparallel alignment; thus no spin alignment can be found that is satisfactory to all interactions: to random bonds $([4,11])$ or random fields $([11,12])$.

To study a different kind of this competition, particularly between ferromagnetic and antiferromagnetic bonds, in our paper, as in [2,3], we have considered an Ising model defined on a tangled chain, in which more bonds have been added to those of pure Ising chain (these bonds provide additional links between spins for additional correlations). Where the chain touches itself (knots), two otherwise distant parts of the original lattice are brought into proximity and coupled by a bond across the chain. The spins placed on the original lattice now have some additional bonds across the chain. In this paper (I) we shall study, using some simple analytical calculations and an iterative algorithm, the bchaviour of the free energy and entropy, particularly in the zero-field and zero-temperature limit, for different configurations of the ferromagnetic tangled chain and different types of additional interactions (ferromagnetic or antiferromagnetic). In the same way, using the same model and a similar iterative algorithm, we shall study in the next paper (II) the behaviour of the magnetization and susceptibility.

Similarly we can follow the same procedure to study the case of an antiterromagnetic tangled chain with ferromagnetic and antiferromagnetic additional interactions.

\section{The model and the transfer matrix method}

Consider ferromagnetic Ising spins placed on a tangled chain with a coupling constant $-J(J>0)$ between the nearest neighbours along the chain and with a coupling constant $-J^{\prime}\left(J^{\prime}>0, J^{\prime}=0\right.$ or $\left.J^{\prime}<0\right)$ across the chain. $A$ s far as the competition effects between different bonds are concerned, the only relevant information that cnters into the IIamiltonian is how spins are coupled. Thus we stretch the tangled chain while allowing those bonds across the chain also to stretch as shown in Fig. 1, where there is represented a "box" (consisting of a tangled part and an untangled one) of the stretched chain. The bonds $J^{\prime}$ represent these stretched "long range" bonds. We suppose that there are $n_{\mathrm{d}}$ of them uniformly distributed along the chain. Between the two opposite ends of each long range bond, there are $n_{1}$ spins. There are $n_{2}$ spins between one end of a long range bond and the beginning of the next long range bond. These parameters of chain configuration are then related to the total number of spins by $N=n_{\mathrm{d}}\left(n_{1}+n_{2}+2\right)$, where the number 2 is to count those spins coupled by the long range bonds. 


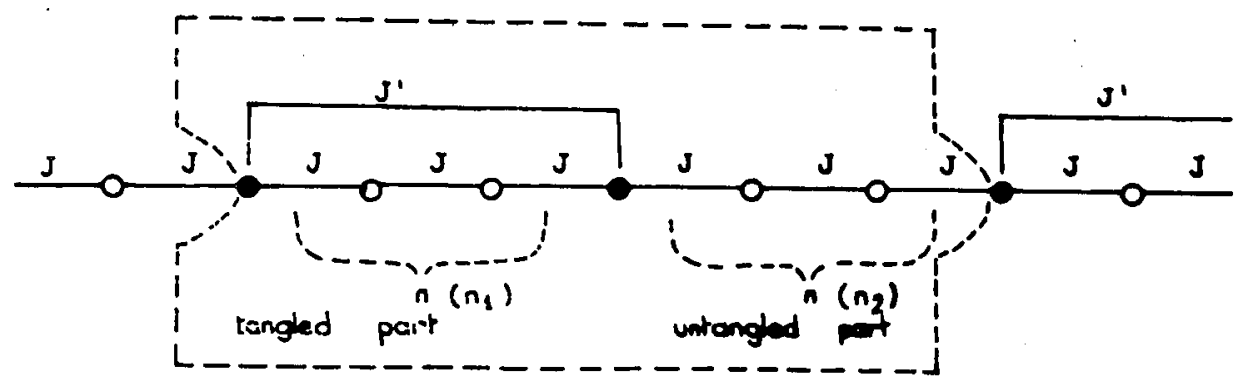

Fig. 1. Schematic presentation of a "box" or a segment of the chain, consisting of a tangled and an untangled part.

For the present model the IIamiltonian of the system is ([3]):

$$
H(S)=H o(S)+H^{\prime}(S)
$$

where

$$
\begin{aligned}
& H o(S)=-J \sum_{\mathrm{nn}} S_{i} S_{j}-B \sum_{i} S_{i}, \\
& H^{\prime}(S)=-J^{\prime} \sum_{\mathrm{dn}} S_{i} S_{j} .
\end{aligned}
$$

$B$ is the magnetic field and $S_{i}= \pm 1$. The pure system IIamiltonian $H o(S)$ represents the coupling of all spins with thir nearest neighbours (nin) along the chain and the "perturbation" term $H^{\prime}(S)$ considers additional bonds between selected pairs of distant neighbours (dn). Not all spins have such a "long range" bond, and therefore this model, which has a kind of bond disorder, is different from the other one-dimensional models which have true long range bonds.

Following $[3,1]$ it is helpful to consider the chain as consisting of many small segments which are tied together at "knots", to take a segment and to treat it in terms of an effective coupling and an effective field only for the two spins, remaining at the opposite ends of this segment. Therefore, for a segment of $(n+2)$ spins, the traced middle part over $S_{2} \ldots S_{n+1}$ leaves for two spins $S_{1}, S_{n+2}$ on the opposite ends of the segment, a temperature dependent constant $c[n]$, an effective field $y[n]$ and an effective coupling $x[n]$, generated from $c[0], y[0], x[0]$ (i.e., from a two-spin segment which has no middle part) by the recurrence relations

$$
\begin{aligned}
& c[n]=2(y[0] y[n-1]+c[0] c[n-1]), \\
& y[n]=2(y[0] x[n-1]+c[0] y[n-1]), \\
& x[n]=2(x[0] x[n-1]+y[0] y[n-1]),
\end{aligned}
$$

with

$$
\begin{aligned}
& c[0]=0.5\left[k \operatorname{ch}(f / t)+k^{-1}\right], \\
& y[0]=0.5 k \operatorname{sh}(f / t), \\
& x[0]=0.5\left[k \operatorname{ch}(f / t)-k^{-1}\right],
\end{aligned}
$$


where $t=\left(k_{\mathrm{B}} T / J\right)\left(k_{\mathrm{B}}\right.$ - the Boltzmann constant and $T$ - the absolute temperature) and $f=(B / J)$ are the reduced temperature and the reduced field, respectively, and $k=\exp (1 / t)$.

The transfer matrix $T$ :

$$
T\left(n_{1}, n_{2}\right)=\left(\begin{array}{ll}
T_{++} & T_{+-} \\
T_{-+} & T_{--}
\end{array}\right)
$$

can be expressed through the matrix multiplication

$$
\boldsymbol{T}\left(n_{1}, n_{2}\right)=\boldsymbol{T P}\left(n_{1}\right) \cdot \boldsymbol{U P}\left(n_{2}\right),
$$

where the first matrix $\boldsymbol{T P}$ on the right represents the tangled part and the second matrix $\boldsymbol{U P}$ the untangled part. Their elements are, respectively

$$
\begin{aligned}
& a\left(n_{1}, k, k^{\prime}, f\right) \equiv a[n 1]=k^{\prime}(c[n 1]+2 y[n 1]+x[n 1]) \equiv k^{\prime} a_{e}, \\
& b\left(n_{1}, k, k^{\prime}, f\right) \equiv b[n 1]=k^{\prime-1}(c[n 1]-x[n 1]) \equiv k^{\prime-1} b_{e}, \\
& d\left(n_{1}, k, k^{\prime}, f\right) \equiv d[n 1]=k^{\prime}(c[n 1]-2 y[n 1]+x[n 1]) \equiv k^{\prime} d_{e},
\end{aligned}
$$

where $k^{\prime}=\exp \left(J^{\prime} / k_{\mathrm{B}} T\right)=\exp (e / t)$ with $e=J^{\prime} / J$, and

$$
\begin{aligned}
& a\left(n_{2}, k, 0, f\right) \equiv a[n 2]=c[n 2]+2 y[n 2]+x[n 2] \equiv a_{0}, \\
& b\left(n_{2}, k, 0, f\right) \equiv b[n 2]=c[n 2]-x[n 2] \equiv b_{0}, \\
& d\left(n_{2}, k, 0, f\right) \equiv d[n 2]=c[n 2]-2 y[n 2]+x[n 2] \equiv d_{0} .
\end{aligned}
$$

From (5)-(7) we obtain

$$
\begin{aligned}
& T_{++} \equiv A=a[n 1] a[n 2]+b[n 1] b[n 2]=k^{\prime} a_{e} a_{0}+k^{\prime-1} b_{e} b_{0}, \\
& T_{+-} \equiv B=a[n 1] b[n 2]+b[n 1] d[n 2]=k^{\prime} a_{e} b_{0}+k^{\prime-1} b_{e} d_{0}, \\
& T_{-+} \equiv C=b[n 1] a[n 2]+d[n 1] b[n 2]=k^{\prime-1} b_{e} a_{0}+k^{\prime} d_{e} b_{0}, \\
& T_{--} \equiv D=b[n 1] b[n 2]+d[n 1] d[n 2]=k^{\prime-1} b_{e} b_{0}+k^{\prime} d_{e} d_{0} .
\end{aligned}
$$

For the larger eigenvalue of the transfer matrix we have

$$
\lambda_{\max } \equiv \lambda_{+}=0.5\left[(A+D)+\sqrt{(A-D)^{2}+4 B C}\right] \text {. }
$$

Since the maximum eigenvalue $\lambda_{\max }$ is cqual to the grand partition function per box (or $n_{1}+n_{2}+2$ sites), in the thermodynamic limit, the thermodynamic potential or the free energy per site is derived as

$$
\frac{F_{N}}{N}=-k_{\mathrm{B}} T \lim _{N \rightarrow \infty}\left[N^{-1} \ln (\lambda)^{N /(n 1+n 2+2)}\right]=-\frac{k_{\mathrm{B}} T}{n_{1}+n_{2}+2} \ln \lambda_{\max } .
$$

Now we shall study, using the main line of this analytical procedure and an iterative algorithm based on that, the behaviour of the free energy and entropy, particularly in the zero-field and zero-temperature limit, for different configurations of the ferromagnetic tangled chain and different types of additional interactions. A lot of work is done to study this behaviour in different models, even and very recently $([11,13])$. 


\section{The free energy}

\subsection{The simple case $\left(n_{1}=n_{2}=1\right)$}

In Sec. 2 we have given only the main lines of the general procedure to calculate the free energy. In this simple case from (3)-(4) we obtain

$$
\begin{aligned}
& c[1]=0.5\left[k^{2} \operatorname{ch}(2 f / t)+2 \operatorname{ch}(f / l)+k^{-2}\right], \\
& y[1]=0.5\left[k^{2} \operatorname{sh}(2 f / t)\right], \\
& x[1]=0.5\left[k^{2} \operatorname{ch}(2 f / t)-2 \operatorname{ch}(f / l)+k^{-2}\right],
\end{aligned}
$$

while from (6)-(7) and (11) we have

$$
\begin{aligned}
& a_{e}=a_{0}=k^{2} \exp (2 f / t)+k^{-2}, \\
& b_{e}=b_{0}=2 \operatorname{ch}(f / t), \\
& d_{e}=d_{0}=k^{2} \exp (-2 f / t)+k^{-2} .
\end{aligned}
$$

Substituting these expressions to (8), the elements of the transfer matrix in this simple case will be

$$
\begin{aligned}
& A=k^{\prime}\left[k^{2} \exp (2 f / t)+k^{-2}\right]^{2}+k^{\prime-1}[2 \operatorname{ch}(f / t)]^{2}, \\
& B=2 \operatorname{ch}(f / t)\left\{k^{\prime}\left[k^{2} \exp (2 f / t)+k^{-2}\right]+k^{\prime-1}\left[k^{2} \exp (-2 f / t)+k^{-2}\right]\right\}, \\
& C=2 \operatorname{ch}(f / t)\left\{k^{\prime-1}\left[k^{2} \exp (2 f / l)+k^{-2}\right]+k^{\prime}\left[k^{2} \exp (-2 f / t)+k^{-2}\right]\right\}, \\
& D=k^{\prime-1}[2 \operatorname{ch}(f / t)]^{2}+k^{\prime}\left[k^{2} \exp (-2 f / t)+k^{-2}\right]^{2} .
\end{aligned}
$$

From (13), using (9) we may study the variation of the free energy per site (10) versus the reduced temperature $t$. To have an idea, one of this diagram $(F-t)$, for the case $f=1$ and different values of interaction ratio $e$, is represented in Fig. 2 . In the case $e=0$ or $J^{\prime}=0$ (i.e., there are no additional bonds) $\lambda_{\max }$ is reduced to $\lambda_{1}^{4}$, where $\lambda_{1}=k \operatorname{ch}(f / t)+\sqrt{k^{2} \operatorname{sh}^{2}(f / t)+k^{-2}}$ is the maximum cigenvalue or the partition function per site of the usual 1D (unperturbed) Ising model with a coupling constant $-J$ of nearest neighbours $([14,15])$.

In the zero-field limit $(f=0)$, from (13) we obtain

$$
\begin{aligned}
& A(0)=D(0)=4 k^{\prime-1}+k^{\prime}\left(k^{2}+k^{-2}\right)^{2}, \\
& B(0)=C(0)=2\left(k^{2}+k^{-2}\right)\left(k^{\prime}+k^{\prime-1}\right),
\end{aligned}
$$

and the maximum eigenvalue is given by

$$
\lambda_{\max }(0)=4 k^{\prime-1}+k^{\prime}\left(k^{2}+k^{-2}\right)^{2}+2\left(k^{2}+k^{-2}\right)\left(k^{\prime}+k^{\prime-1}\right) .
$$

In the zero-temperature limit $(t \rightarrow 0)$ we have

$$
\begin{aligned}
& k=\exp (1 / t) \rightarrow \infty, \quad k^{-1}=\exp (-1 / l) \rightarrow 0(J>0), \\
& k^{\prime}=\exp (e / t) \rightarrow \infty(e>0), \quad k^{\prime}=1(e=0), \quad k^{\prime}=\exp (e / t) \rightarrow 0(e<0) .
\end{aligned}
$$




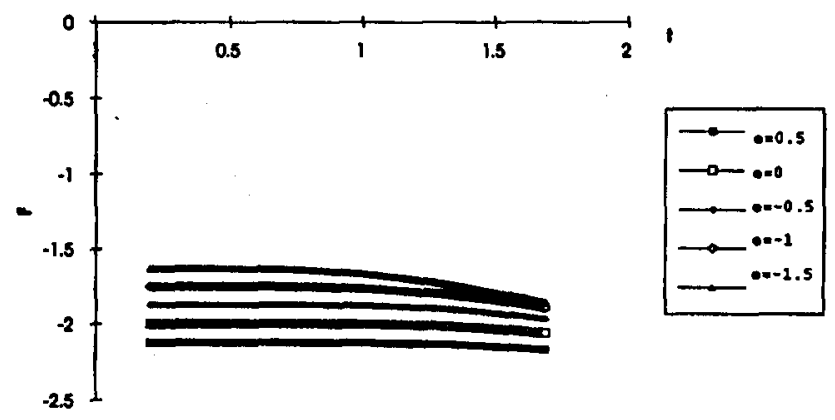

Fig. 2. Free energy $F$ as a function of reduced temperature $t(f=1$ and $e=0.5,0$, $-0.5,-1,-1.5)$.

Considering "the highest power" tcrm in the expression of the zero-field frce energy (when $t \rightarrow 0$ ) we obtain

$$
\begin{aligned}
& F=\frac{F_{N}}{N J} \approx-[(4+e) / 4](e>-1), \\
& F \approx-(1 / 4)(3+t \ln 3) \sim-0.75(e=-1), \\
& F \approx-(1 / 4)[(2-e)+t \ln 2] \sim-[(2-e) / 4](e<-1) .
\end{aligned}
$$

Therefore, the reduced zero-temperature internal energy per spin, for the "transition" value $e=-1$ is $U(0)=-0.75$ (see [16]). In Fig. 3 there are represented, as illustration, the numerical variations of the free encrgy versus the temperature $t$, in the zero-field limit. For $t=0.08$ (the lowest temperature accessible in this case) the value of $F$ is -0.77 (while the value of the term $-(t \ln 3) / 4$ is $\approx-0.02$ ).

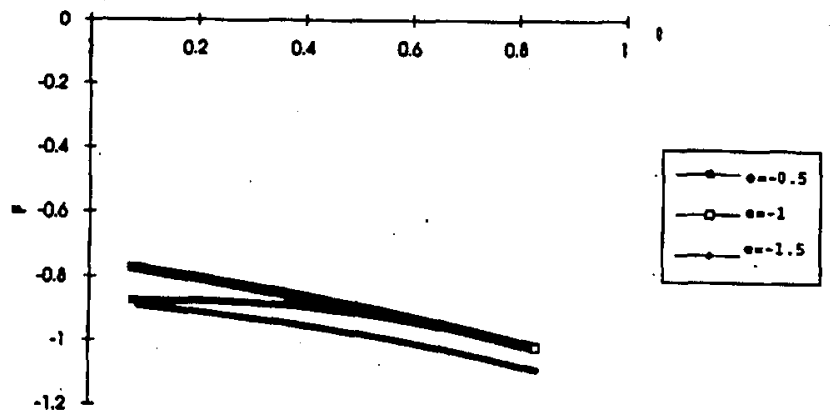

Fig. 3. $F$ as a function of $t(f=0$ and $\dot{e}=-0.5,-1,-1.5)$. 


\subsection{The general. case $\left(n_{1} \neq n_{2}\right)$}

The simplicity of our model has allowed us to perform sume analytical calculations for the simple configurations (as $n_{1}=n_{2}=1, n_{1}=n_{2}=2$, etc.) which are very instructive. IIowever, even for such a simple model it is very complicated to push analytic calculations very far, particularly in the case of a non-zero magnetic field. Numerical calculations have also been done to supplement the analytic work. Therefore, using an itcrative algorithm, based on the main lines of the general procedure given in Sec. 2, it is possible and very suitable to study the variation of $F$ versus $t$ for different chain configurations (for different valucs of $n_{1}$ and $n_{2}$ ), for different values of interaction ratio $e$ and different values of field $f$. For illustration, one of $(F-t)$ diagrams in the zero-field limit $(f=0)$ is represented in Fig. 4. Also, some of the approximate estimations of the zero-field and the zero-temperature internal energy, limited in accuracy by the value of the lowest temperature accessible numerically, for the "transition" interaction parameter $e=-1$, are as follows:

$$
\begin{array}{ll}
n_{1}=2, n_{2}=1,-0.83, & n_{1}=2, n_{2}=2,-0.86, \\
n_{1}=3, n_{2}=1,-0.86, & n_{1}=3, n_{2}=2,-0.89, \\
n_{1}=4, n_{2}=1,-0.89, & n_{1}=4, n_{2}=2,-0.91, \\
n_{1}=5, n_{2}=1,-0.91, & n_{1}=5, n_{2}=2,-0.93 .
\end{array}
$$

It is clearly visible that an important feature is the total number of bonds per box. Also the highest value of the internal energy $(f=0$ and $t=0)$ is for the configuration $n_{1}=n_{2}=1$, wliere the number of additional bonds is the biggest (one $J^{\prime}$ for four $J$ per each box) except for the cases: $n_{1}=1, n_{2}=0$ or $n_{1}=0$, $n_{2}=1$ (one $J^{\prime}$ for three $J$ per each box) or $n_{1}=0, n_{2}=0$ (which corresponds to an Ising model with alternate interactions $J_{1}=J+J^{\prime}$ and $J_{2}=J$ ). Thus, among the cases with $n_{1} \neq 0, n_{2} \neq 0$, for the configuration $n_{1}=n_{2}=1$ we have the most powerful competition effect between ferromagnetic and antiferromagnetic bonds.

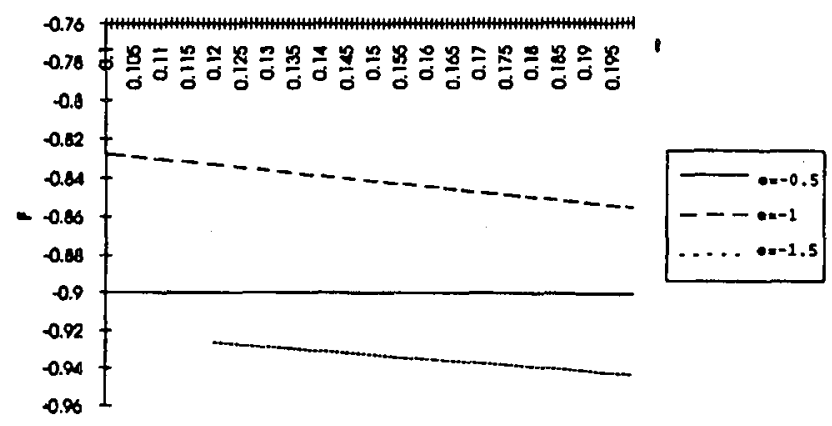

Fig. 4. (F-l) diagram for the configuration $n_{1}=2, n_{2}=1(f=0$ and $e=-0.5,-1$, $-1.5)$. 


\section{The entropy \\ 4.1. The simple case $\left(n_{1}=n_{2}=1\right)$}

From the usual thermodynamic relation the reduced entropy per spin is given by

$$
\begin{aligned}
S=\frac{S_{N}}{N J}=\left[1 /\left(n_{1}+n_{2}+2\right)\right] \ln \lambda_{\max }+\left[t /\left(n_{1}+n_{2}+2\right)\right] \\
\times\left[\left(\frac{\partial \lambda_{\max }}{\partial t}\right) / \lambda_{\max }\right]
\end{aligned}
$$

which in the case $e=0\left(J^{\prime}=0\right)$ is reduced to the expression of the entropy for a $1 D$ Ising model with nearest neighbour interactions. From (16) and the concrete expression of the first derivative of $\lambda_{\max }$ we may study the variation of the entropy against the temperature for different cases. One of these $(S-t)$ diagrams is represented in Fig. 5. In Fig. 6 these variations are represented in the zero-field limit.

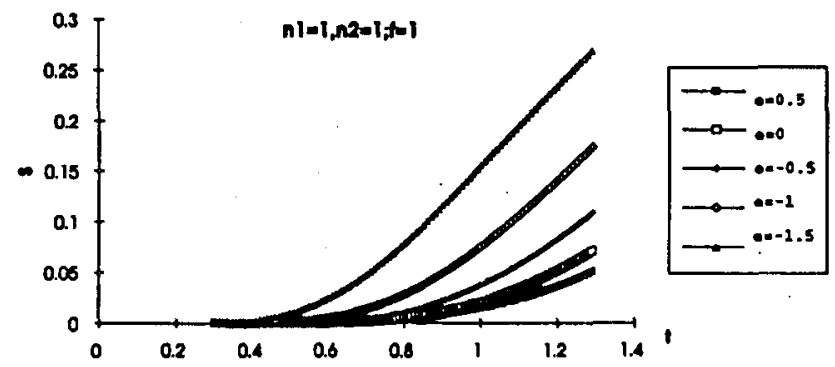

Fig. 5. Entropy $S$ against reduced temperature $t(f=1$ and $e=0.5,0,-0.5,-1,-1.5)$.

Considering "the highest power" term in the expression of the zero-field entropy, when $t \rightarrow 0$, we find that

$$
\begin{aligned}
& S \rightarrow 0(e>-1), \\
& S \rightarrow[(\ln 3) / 4] \sim 0.27(e=-1), \\
& S \rightarrow[(\ln 2) / 4] \sim 0.17(e<-1) .
\end{aligned}
$$

These results (for $e \leq-1$ ) indicate the existence of non-zcro entropy at zero temperature. In Fig. 7 we have illustrated the case $e=-1$, using numerical calculations. Because of the restriclions on the lowest temperature that can be reached, only temperatures $T \geq 0.09 \mathrm{~J}$ are accessible. (In the region $0.075 \leq t \leq 0.09$ "the fluctuations" are considerable (the small diagram in Fig. 7) and the values below 0.075 are not accessible.) A zero-temperature entropy, different from zero, is obtained many ycars ago from Puma and Fernandez [17] for the random field Ising model, but also very recently for the frustrated quantum spin $\frac{1}{2}$ chain [11]. 


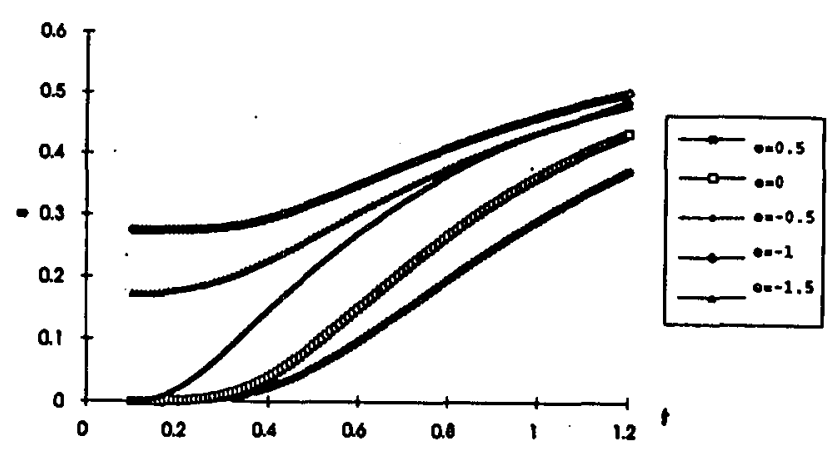

Fig. 6. Entropy $S$ as a function of reduced temperature $t$ in the zero-field limit $(f=0$ and $e=0.5,0,-0.5,-1,-1.5)$.

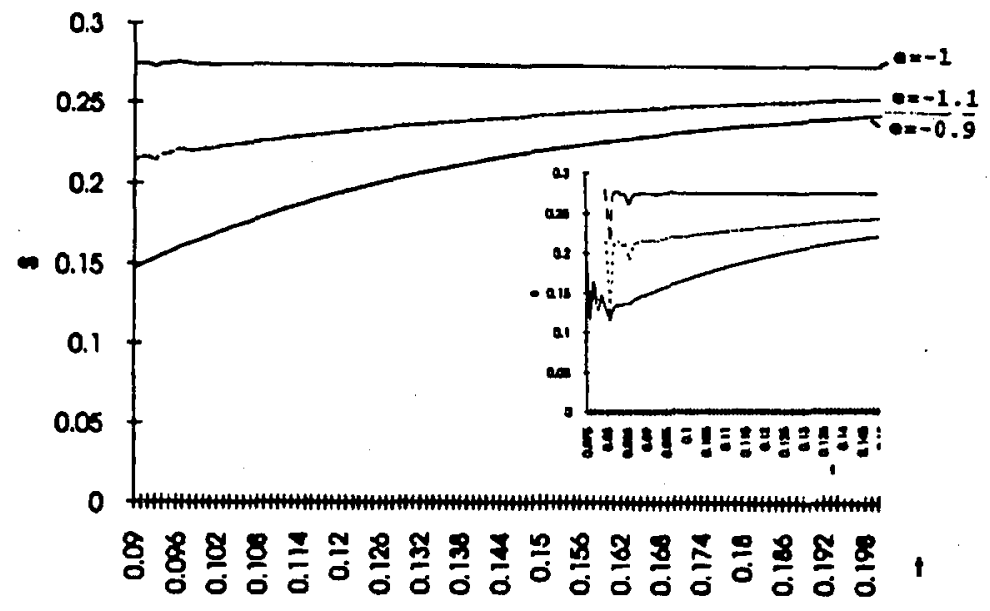

Fig. 7. Entropy $S$ against the reduced temperature for very small values of $t$ ( $f=0$ and $e=-0.9,-1,-1.1$. The value of the zero-field cntropy is finite for $e \leq-1$ when $t \rightarrow 0$.

\subsection{The general case $\left(n_{1} \neq n_{2}\right)$}

To obtain numerically the variation of the entropy versus the temperature, for different chain configurations and different values of $e$ and $f$, we have to complete the iterative algorithm, used for the frce energy, by the first derivatives (denoted with') related to the temperature $t$ of the following groups of quantities:

$$
\begin{aligned}
& c^{\prime}[0]=-\left(0.5 / t^{2}\right)\left[k(\operatorname{ch}(f / l)+f \operatorname{sh}(f / t))-k^{-1}\right], \\
& y^{\prime}[0]=-\left(0.5 / t^{2}\right)[k(\operatorname{sh}(f / l)+f \operatorname{ch}(f / l))], \\
& x^{\prime}[0]=-\left(0.5 / t^{2}\right)\left[k(\operatorname{ch}(f / t)+f \operatorname{sh}(f / l))+k^{-1}\right],
\end{aligned}
$$




$$
\begin{aligned}
& c^{\prime}[n]=2\left(y^{\prime}[0] y[n-1]+y[0] y^{\prime}[n-1]+c^{\prime}[0] c[n-1]+c[0] c^{\prime}[n-1]\right), \\
& y^{\prime}[n]=2\left(y^{\prime}[0] x[n-1]+y[0] x^{\prime}[n-1]+c^{\prime}[0] y[n-1]+c[0] y^{\prime}[n-1]\right) \text {, } \\
& x^{\prime}[n]=2\left(x^{\prime}[0] x[n-1]+x[0] x^{\prime}[n-1]+y^{\prime}[0] y[n-1]+y[0] y^{\prime}[n-1]\right), \\
& a_{0}^{\prime}=c^{\prime}[n 2]+2 y^{\prime}[n 2]+x^{\prime}[n 2], \\
& b_{0}^{\prime}=c^{\prime}[n 2]-x^{\prime}[n 2] \text {, } \\
& d_{0}^{\prime}=c^{\prime}[n 2]-2 y^{\prime}[n 2]+x^{\prime}[n 2] \text {, } \\
& a_{e}^{\prime}=c^{\prime}[n 1]+2 y^{\prime}[n 1]+x^{\prime}[n 1] \\
& b_{e}^{\prime}=c^{\prime}[n 1]-x^{\prime}[n 1] \text {, } \\
& d_{e}^{\prime}=c^{\prime}[n 1]-2 y^{\prime}[n 1]+x^{\prime}[n 1] \text {, } \\
& A^{\prime}=\exp (e / t)\left(a_{0} a_{e}^{\prime}+a_{0}^{\prime} a_{e}\right)+\exp (-e / t)\left(b_{0} b_{e}^{\prime}+b_{0}^{\prime} b_{e}\right) \\
& -\left(e / \iota^{2}\right)\left[\exp (e / l) a_{0} a_{e}-\exp (-c / l) b_{0} b_{e}\right] \\
& B^{\prime}=\exp (e / t)\left(a_{e} b_{0}^{\prime}+a_{e}^{\prime} b_{0}\right)+\operatorname{cxp}(-e / l)\left(b_{e} d_{0}^{\prime}+b_{e}^{\prime} d_{0}\right) \\
& -\left(e / t^{2}\right)\left[\exp (e / l) a_{e} b_{0}-\exp (-e / t) b_{e} d_{0}\right], \\
& C^{\prime}=\exp (-e / \iota)\left(b_{e} a_{0}^{\prime}+b_{e}^{\prime} a_{0}\right)+\exp (e / l)\left(d_{e} b_{0}^{\prime}+d_{e}^{\prime} b_{0}\right) \\
& +\left(e / l^{2}\right)\left[\exp (-e / l) a_{0} b_{e}-\operatorname{cxp}(e / l) d_{e} b_{0}\right], \\
& D^{\prime}=\exp (-e / t)\left(b_{e} b_{0}^{\prime}+b_{e}^{\prime} b_{0}\right)+\exp (e / l)\left(d_{e} d_{0}^{\prime}+d_{e}^{\prime} d_{0}\right) \\
& +\left(e / \iota^{2}\right)\left[\exp (-e / l) b_{0} b_{e}-\exp (e / l) d_{0} d_{e}\right],
\end{aligned}
$$

and also

$$
\begin{aligned}
\lambda_{\max }^{\prime} & =0.5\left\{A^{\prime}+D^{\prime}+\left[(A-D)\left(A^{\prime}-D^{\prime}\right)\right.\right. \\
& \left.\left.+2\left(B^{\prime} C+B C^{\prime}\right)\right] / \sqrt{(A-D)^{2}+4 B C}\right\} .
\end{aligned}
$$

Using an iterative algorithm based on these recurrence relations we can study the variations of entropy $S$ against temperature $t$ for different combinations of $n_{1}, n_{2}, e$ and $f$. In Fig. 8 there is represented one of these $(S-t)$ diagrams for the case $e=-1, f=0$ and some combinations of $\left(n_{1}, n_{2}\right)$. Also, it is possible to estimate numerically the values of the zero-temperature entropy for $e=-1$ and different chain configurations, with an accuracy limited by the lowest accessible temperature. Some of these $S(0)$ values, in the zcro-field condition, are

$$
\begin{array}{ll}
n_{1}=2, n_{2}=1, \approx 0.277, & n_{1}=2, n_{2}=2, \approx 0.231, \\
n_{1}=3, n_{2}=1, \approx 0.268, & n_{1}=3, n_{2}=2, \approx 0.230, \\
n_{1}=4, n_{2}=1, \approx 0.256, & n_{1}=4, n_{2}=2, \approx 0.224 .
\end{array}
$$




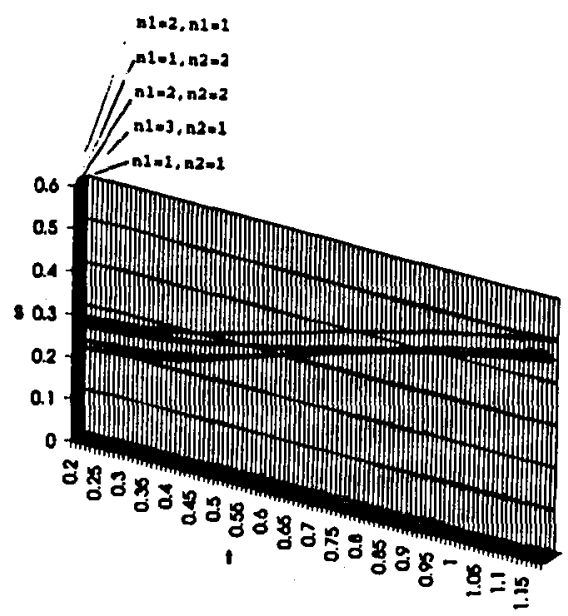

Fig. 8. Entropy $S$ as a function of temperature $t$ for different combinations of $\left(n_{1}, n_{2}\right)$ ( $f=0$ and $e=-1$ ). The ends of curves on vertical columns are placed following this order of configuration: $\left(n_{1}=2, n_{2}=1\right) ;\left(n_{1}=1, n_{2}=2\right) ;\left(n_{1}=2, n_{2}=2\right)$; $\left(n_{1}=3, n_{2}=1\right) ;\left(n_{1}=1, n_{2}=1\right)$.

\section{Conclusions}

In this paper we have considered an Ising model on a tangled chain to understand the competition between ferromagnetic bonds of the usual chain and additional bonds of ferromagnetic or antiferromagnetic types, manifested in the behaviour of the free energy and entropy. Using a modified transfer matrix method, some simple analytical calculations and an iterative algorithm, we have studied in detail this behaviour, particularly in the zero-field and zero-temperature limit, for different configurations of composed parts (boxes) in the ferromagnetic tangled chain and different types of additional interactions. We have found that this behaviour is different in different regions of the interaction ratio (additional interaction $J^{\prime}$ /usual $n n$ interaction $J$ ) and the condition $J=-J^{\prime}$, between the ferromagnetic interaction $J$ along the chain and the antiferromagnetic interaction $J^{\prime}$ across the chain, plays a role of a "transition region" condition for this behaviour. Our results indicate also the existence of non-zero entropy at zero temperature. This fact "speaks" clearly for presence of a type of frustration in the tangled chain model, which has a kind of bond disorder. It is rather difficult to interpret these results in terms of uneven effective local temperatures, considering that a perturbation in local interaction may be regarded as a perturbation in local temperatures.

In the same way we can study also the case of an antiferromagnetic tangled chain with ferromagnetic or antiferromagnetic additional interactions.

A very important feature is also the behaviour of the magnetization and susceptibility, particularly in the above mentioned limits. Our results about this behaviour, by using a similar iterative algorithm, will be represented in the next paper (II). 


\section{Acknowledgments}

The author is very grateful to Prof. R.B. Stinchcombe (Oxford) for his stimulating interest and very helpful orientation at the beginning of this work. IIe would like also to thank Prof. Abdus Salam, the International Atomic Encrgy Agency and UNESCO for the hospitality at the International Centre for Theoretical Physics, Trieste.

\section{References}

[1] R. Mejdani, preprint IC/93/67, ICTP-Trieste 1993.

[2] J.C. Lee, Physica A 160, 503 (1989).

[3] J.C. Lee, J.M. Stephens, L.R. Mead, R.F. Joyce, Physica A 167, 749 (1990).

[4] K. Binder, A.P. Young, Rev. Mod. Phys. 58, 801 (1986).

[5] M. Mezard, G. Parisi, M.A. Virasoro, Spin Class Theory and Beyond, Lectures Notes in Physics, Vol. 9, World Scientific, Singapore 1987.

[6] K.H. Fisher, J.A. Hertz, Spin Glasses, Cambridge University Press, Cambridge 1991.

[7] R.A. Cowley, R.J. Birgencau, G. Shirane, Physica A 140, 285 (1986).

[8] H.H. Chen, S. Ma, J. Slat. Phys. 20, 717 (1982).

[9] S. Masui, A.E. Jacobs, C. Wicentowich, B.W. Southern, J. Phys. A, Malh. Gen. 26, 25 (1993).

[10] G. Toulouse, Commun. Phys. 2, 115 (1977).

[11] P. Reed, J. Phys. A, Math. Gen. 25, 5861 (1992).

[12] D.S. Fisher, Phys. Rev. B 31, 7233 (1984).

[13] L. Yi, G. Buttner, K.D. Usadel, K.L. Yao, Phys. Rev. B 47, 254 (1993).

[14] M. Toda, R. Kubo, N. Saito, Slalistical Physics I, Springer-Verlag, Berlin 1992, Ch. 4.

[15] C.J. Thompson, Malhematical Statistical Mechanics, Princeton University Press, Princeton 1979, Ch. 5.

[16] G. Parisi, J. Phys. A, Math. Gen. 13, L115 (1980).

[17] M. Puma, J.F. Fernandez, Phys. Rev. B 18, 1391 (1978). 